\title{
CARTAN CONNECTIONS AND NATURAL AND PROJECTIVELY EQUIVARIANT QUANTIZATIONS
}

\author{
P. MATHONET AND F. RADOUX
}

\begin{abstract}
In this paper, we analyse the question of existence of a natural and projectively equivariant symbol calculus, using the theory of projective Cartan connections. We establish a close relationship between the existence of such a natural symbol calculus and the existence of an $\operatorname{sl}(m+1, \mathbb{R})$ - equivariant calculus over $\mathbb{R}^{m}$ in the sense of 15 1. Moreover we show that the formulae that hold in the non-critical situation over $\mathbb{R}^{m}$ for the $s l(m+1, \mathbb{R})$ - equivariant calculus can be directly generalized to an arbitrary manifold by simply replacing the partial derivatives by invariant differentiations with respect to a Cartan connection.
\end{abstract}

\section{INTRODUCTION}

A quantization procedure can be roughly defined as a linear bijection from the space of classical observables to a space of differential operators acting on wave functions (at least in the framework of geometric quantization, see 24]).

In our setting, the space $\mathcal{S}(M)$ of observables (also called Symbols) is made of smooth functions on the cotangent bundle $T^{*} M$ of a manifold $M$, that are polynomial along the fibres. The space $\mathcal{D}_{\lambda}(M)$ of differential operators consists of operators acting on $\lambda$-densities over $M$.

It is known that there is no natural quantization procedure. In other words, the spaces of symbols and of differential operators are not isomorphic as representations of $\operatorname{Diff}(M)$.

The idea of equivariant quantization, introduced by P. Lecomte and V. Ovsienko in [15] is to reduce the group of (local) diffeomorphisms under consideration.

They studied the case of the projective group $P G L(m+1, \mathbb{R})$ acting locally on the manifold $M=\mathbb{R}^{m}$ by linear fractional transformations. They showed that the spaces of symbols and of differential operators are canonically isomorphic as representations of $P G L(m+1, \mathbb{R}$ ) (or its Lie algebra $s l(m+1, \mathbb{R}))$. In other words, they showed that there exists a unique projectively equivariant quantization.

Date: October 25, 2018.

University of Liège, Institute of mathematics, Grande Traverse, 12 - B37, B-4000 Liège, Belgium email : P.Mathonet@ulg.ac.be, F.Radoux@ulg.ac.be MSC : 53B10, 53C10, 22E46. 
Independently of quantization purposes, the equivariant quantization map proved to be a useful tool in the study of the spaces of differential operators. Indeed, the inverse of such a map, called projectively equivariant symbol map, is an equivariant bijection from the filtered space $\mathcal{D}_{\lambda}(M)$ to its associated graded space $\mathcal{S}(M)$ and allows to develop an equivariant symbol calculus.

In 9 , the authors studied the spaces $\mathcal{D}_{\lambda \mu}\left(\mathbb{R}^{m}\right)$ of differential operators transforming $\lambda$-densities into $\mu$-densities and their associated graded spaces $\mathcal{S}_{\delta}$. They showed the existence and uniqueness of a projectively equivariant quantization, provided the shift value $\delta=\mu-\lambda$ does not belong to a set of critical values.

A first example of projectively equivariant symbol calculus for differential operators acting on tensor fields was given in [1].

At that point, all these results were dealing with a manifold endowed with a flat projective structure. In [4, 5], S. Bouarroudj showed that the formula for the projectively equivariant quantization for differential operators of order two and three could be expressed using a torsion-free linear connection, in such a way that it only depends on the projective class of the connection.

In [17, P. Lecomte conjectured the existence of a quantization procedure

$$
Q: \mathcal{S}_{\delta}(M) \rightarrow \mathcal{D}_{\lambda \mu}(M)
$$

depending on a torsion-free linear connection, that would be natural (in all arguments) and that would remain invariant under a projective change of connection.

The existence of such a Natural and equivariant quantization procedure was proved by M. Bordemann in [3], using the notion of Thomas-Whitehead connection associated to a projective class of connections (see 21, 23, 12, 20, 19 for a discussion of Thomas-Whitehead connections). His construction was later adapted by S. Hansoul in [1] in order to deal with differential operators acting on forms, thus extending the results of 1 .

In [18, we analysed the existence problem for a natural and projectively equivariant quantization using the theory of projective Cartan connections. We obtained an explicit formula for the natural and projectively equivariant quantization in terms of the normal Cartan connection associated to a projective class of linear connections.

To our astonishment, it turned out that this explicit formula is nothing but the formula given in [9] for the flat case, up to replacement of the partial derivatives by invariant differentiations with respect to the Cartan connection. In particular, we showed that the natural and projectively equivariant quantization exists if and only if the $\operatorname{sl}(m+1, \mathbb{R})$-equivariant quantization exists in the flat case.

Recently, in her thesis, S. Hansoul [10] showed how to generalize the method given by M. Bordemann in order to solve the problem of existence of a natural and projectively equivariant symbol calculus for differential operators acting on arbitrary tensor fields. 
In this paper, we analyse this question with the help of Cartan projective connections. The advantage of our method is that it provides a direct generalization of the formulae that can be written down in the context of projectively equivariant symbol calculus over $\mathbb{R}^{m}$ (in the non-critical situation), simply by replacing the partial derivatives by invariant differentiations with respect to a Cartan connection. It also provides a direct link between the existence of the $s l(m+1, \mathbb{R})$-equivariant quantization over $M=\mathbb{R}^{m}$ and the existence of a natural and projectively equivariant quantization over an arbitrary manifold.

\section{Problem Setting}

In this section, we will describe the definitions of the spaces of differential operators acting on tensor fields and of their corresponding spaces of symbols. Then we will set the problem of existence of projectively equivariant natural symbol calculi.

2.1. Tensor fields. Let $M$ be a smooth manifold of dimension $m \geq 2$. The arguments of the differential operators that we will consider are sections of vector bundles associated to the linear frame bundle. We consider irreducible representations of the group $G L(m, \mathbb{R})$ defined as follows : let $\left(V, \rho_{D}\right)$ be the representation of $G L(m, \mathbb{R})$ corresponding to a Young diagram $Y_{D}$ of depth $n<m$. Fix $\lambda \in \mathbb{R}$ and $z \in \mathbb{Z}$ and set

$$
\rho(A) u=|\operatorname{det}(A)|^{\lambda}(\operatorname{det}(A))^{z} \rho_{D}(A) u,
$$

for all $A \in G L(m, \mathbb{R})$, and $u \in V$.

If $(V, \rho)$ is such a representation, we denote by $V(M)$ the vector bundle

$$
P^{1} M \times_{\rho} V .
$$

We denote by $\mathcal{V}(M)$ the space of smooth sections of $V(M)$. This space can be identified with the space $C^{\infty}\left(P^{1} M, V\right)_{G L(m, \mathbb{R})}$ of functions $f$ such that

$$
f(u A)=\rho\left(A^{-1}\right) f(u) \quad \forall u \in P^{1} M, \forall A \in G L(m, \mathbb{R}) .
$$

Since $V(M) \rightarrow M$ is associated to $P^{1} M$, there are natural actions of $\operatorname{Diff}(M)$ and of $\operatorname{Vect}(M)$ on $\mathcal{V}(M)$.

2.2. Differential operators and symbols. If $\left(V_{1}, \rho_{1}\right)$ and $\left(V_{2}, \rho_{2}\right)$ are representations of $G L(m, \mathbb{R})$, we denote by $\mathcal{D}\left(\mathcal{V}_{1}(M), \mathcal{V}_{2}(M)\right.$ ) (or simply by $\mathcal{D}(M)$ if there is no risk of confusion) the space of linear differential operators from $\mathcal{V}_{1}(M)$ to $\mathcal{V}_{2}(M)$. The actions of $\operatorname{Vect}(M)$ and $\operatorname{Diff}(M)$ are induced by their actions on $\mathcal{V}_{1}(M)$ and $\mathcal{V}_{2}(M)$ : one has

$$
(\phi \cdot D)(f)=\phi \cdot\left(D\left(\phi^{-1} \cdot f\right)\right), \quad \forall f \in \mathcal{V}_{1}(M), D \in \mathcal{D}(M) \text {, and } \phi \in \operatorname{Diff}(M) .
$$

The space $\mathcal{D}(M)$ is filtered by the order of differential operators. We denote by $\mathcal{D}^{k}(M)$ the space of differential operators of order at most $k$. It is wellknown that this filtration is preserved by the action of local diffeomorphisms. 
The space of symbols, which we will denote by $\mathcal{S}_{V_{1}, V_{2}}(M)$ or simply by $\mathcal{S}(M)$, is then the graded space associated to $\mathcal{D}(M)$.

We denote by $S_{V_{1}, V_{2}}^{l}$ the vector space $S^{l} \mathbb{R}^{m} \otimes V_{1}^{*} \otimes V_{2}$. There is a natural representation $\rho$ of $G L(m, \mathbb{R})$ on this space (the representation of $G L(m, \mathbb{R})$ on symmetric tensors is the natural one). We then denote by $S_{V_{1}, V_{2}}^{l}(M) \rightarrow$ $M$ the vector bundle

$$
P^{1} M \times{ }_{\rho} S_{V_{1}, V_{2}}^{l} \rightarrow M,
$$

and by $\mathcal{S}_{V_{1}, V_{2}}^{l}(M)$ the space of smooth sections of $S_{V_{1}, V_{2}}^{l}(M) \rightarrow M$, that is, the space $C^{\infty}\left(P^{1} M, S_{V_{1}, V_{2}}^{l}\right)_{G L(m, \mathbb{R})}$.

Then the principal symbol operator $\sigma: \mathcal{D}^{l}(M) \rightarrow \mathcal{S}_{V_{1}, V_{2}}^{l}(M)$ commutes with the action of diffeomorphisms and is a bijection from the quotient space $\mathcal{D}^{l}(M) / \mathcal{D}^{l-1}(M)$ to $\mathcal{S}_{V_{1}, V_{2}}^{l}(M)$. Hence the space of symbols is nothing but

$$
\mathcal{S}(M)=\bigoplus_{l=0}^{\infty} \mathcal{S}_{V_{1}, V_{2}}^{l}(M),
$$

endowed with the classical actions of $\operatorname{Diff}(M)$ and of $\operatorname{Vect}(M)$.

2.3. Projective equivalence of connections. We denote by $\mathcal{C}_{M}$ the space of torsion-free linear connections on $M$. Two such connections are Projectively equivalent if they define the same geodesics up to parametrization. In algebraic terms, two connections are projectively equivalent if there exists a one form $\alpha$ on $M$ such that their associated covariant derivatives $\nabla$ and $\nabla^{\prime}$ fulfil the relation

$$
\nabla_{X}^{\prime} Y=\nabla_{X} Y+\alpha(X) Y+\alpha(Y) X .
$$

This formulation was given by H. Weyl in [22]

2.4. Natural and equivariant symbol calculus. A (generalized) quantization on $M$ is a linear bijection $Q_{M}$ from the space of symbols $\mathcal{S}_{V_{1}, V_{2}}(M)$ to the space of differential operators $\mathcal{D}\left(\mathcal{V}_{1}(M), \mathcal{V}_{2}(M)\right)$ such that

$$
\sigma\left(Q_{M}(T)\right)=T, \forall T \in \mathcal{S}_{V_{1}, V_{2}}^{k}(M), \forall k \in \mathbb{N} .
$$

The inverse of $Q_{M}$, denoted by $s_{M}$ is a symbol map.

Roughly speaking, a natural quantization map is a quantization map which depends on a torsion free linear connection $\nabla$ and commutes with the action of diffeomorphisms (on all arguments). More precisely, a natural quantization map is a collection of quantizations $Q_{M}$ (defined for every manifold $M$ ) depending on a torsion free linear connection on $M$ such that

(1) For all torsion free linear connections on $M, Q_{M}(\nabla)$ is a quantization map,

(2) If $\phi$ is a local diffeomorphism from $M$ to $N$, then one has

$$
Q_{M}\left(\phi^{*} \nabla\right)\left(\phi^{*} T\right)=\phi^{*}\left(Q_{N}(\nabla)(T)\right), \quad \forall \nabla \in \mathcal{C}(N), T \in \mathcal{S}(N)
$$


Such a natural quantization map is projectively equivariant if one has

$$
Q_{M}(\nabla)=Q_{M}\left(\nabla^{\prime}\right)
$$

whenever $\nabla$ and $\nabla^{\prime}$ are projectively equivalent torsion free linear connections on $M$.

\section{THE FLAT CASE}

Our goal in the present paper is to show the relationship between the existence of a natural and projectively equivariant symbol calculus and the existence of an $\operatorname{sl}(m+1, \mathbb{R})$-equivariant symbol calculus in the sense of [15].

In this section, we will briefly recall the problems addressed in the framework of projectively equivariant quantizations over vector spaces and outline the methods used in [8] and 2]. However, we will generalize the tools of these papers and present them in an more algebraic and coordinate-free fashion.

3.1. Tensor fields, symbols and differential operators. These objects were defined in section 2, but when $M$ is the Euclidean space $\mathbb{R}^{m}$, we make the following identifications :

$$
\begin{aligned}
& \mathcal{V}_{1}\left(\mathbb{R}^{m}\right) \cong C^{\infty}\left(\mathbb{R}^{m}, V_{1}\right), \\
& \mathcal{S}_{V_{1}, V_{2}}^{k}\left(\mathbb{R}^{m}\right) \cong C^{\infty}\left(\mathbb{R}^{m}, S_{V_{1}, V_{2}}^{k}\right) .
\end{aligned}
$$

The Lie algebra $\operatorname{Vect}\left(\mathbb{R}^{m}\right)$ acts on these spaces in a well-known manner : one has

$$
\left(L_{X} T\right)(x)=X . T(x)-\rho_{*}\left(D_{x} X\right) T(x)
$$

for every $X \in \operatorname{Vect}\left(\mathbb{R}^{m}\right)$ and any symbol $T$.

The space $\mathcal{D}\left(\mathbb{R}^{m}\right)$ of differential operators is equipped with the Lie derivative $\mathcal{L}$ given by the commutator.

3.2. The projective algebra of vector fields. Consider the projective group $G=P G L(m+1, \mathbb{R})=G L(m+1, \mathbb{R}) / \mathbb{R}_{0}$ Id. Its Lie algebra $\mathfrak{g}=$ $g l(m+1, \mathbb{R}) / \mathbb{R} I d$ is isomorphic to $\operatorname{sl}(m+1, \mathbb{R})$ and decomposes into a direct sum of subalgebras

$$
\mathfrak{g}=\mathfrak{g}_{-1} \oplus \mathfrak{g}_{0} \oplus \mathfrak{g}_{1}=\mathbb{R}^{m} \oplus \operatorname{gl}(m, \mathbb{R}) \oplus \mathbb{R}^{m *} .
$$

The isomorphism is given explicitly by

$$
\psi: g l(m+1, \mathbb{R}) / \mathbb{R I d} \rightarrow \mathfrak{g}_{-1} \oplus \mathfrak{g}_{0} \oplus \mathfrak{g}_{1}:\left[\left(\begin{array}{cc}
A & v \\
\xi & a
\end{array}\right)\right] \mapsto(v, A-a I d, \xi) .
$$

The group $G=P G L(m+1, \mathbb{R})$ acts on $\mathbb{R} P^{m}$. Since $\mathbb{R}^{m}$ can be seen as the open set of $\mathbb{R} P^{m}$ of equation $x^{m+1}=1$, there is a local action of $G$ on $\mathbb{R}^{m}$. The vector fields associated to this action are given by

$$
\left\{\begin{array}{ccc}
X_{x}^{h} & = & -h \quad \text { if } h \in \mathfrak{g}_{-1} \\
X_{x}^{h} & = & -[h, x] \quad \text { if } h \in \mathfrak{g}_{0} \\
X_{x}^{h} & = & -\frac{1}{2}[[h, x], x] \quad \text { if } h \in \mathfrak{g}_{1}
\end{array},\right.
$$

where $x \in \mathfrak{g}_{-1} \cong \mathbb{R}^{m}$. These vector fields define a subalgebra of $\operatorname{Vect}\left(\mathbb{R}^{m}\right)$, which is isomorphic to $\operatorname{sl}(m+1, \mathbb{R})$. 
Finally, it will be interesting for our computations to recall that the subalgebra $\mathfrak{g}_{0}$ is reductive and decomposes as

$$
\mathfrak{g}_{0}=\mathfrak{h}_{0} \oplus \mathbb{R} \mathcal{E}
$$

where $\mathfrak{h}_{0}$ is (isomorphic to) $s l(m, \mathbb{R})$ and where the Euler element $\mathcal{E}$ is defined by $\left.\operatorname{ad}(\mathcal{E})\right|_{\mathfrak{g}_{-1}}=-I d$.

3.3. Projectively equivariant quantizations. A projectively equivariant quantization (in the sense of [․ [15]) is a quantization

$$
Q: \mathcal{S}_{V_{1}, V_{2}}\left(\mathbb{R}^{m}\right) \rightarrow \mathcal{D}\left(\mathbb{R}^{m}\right)
$$

such that, for every $X^{h} \in \operatorname{sl}(m+1, \mathbb{R})$, one has

$$
\mathcal{L}_{X^{h}} \circ Q=Q \circ L_{X^{h}} .
$$

The existence and uniqueness of such quantizations were discussed in [15. 9, 16. for differential operators acting on densities and in [1] for differential operators acting on forms.

Let us recall a first relationship, already mentioned in [16], between natural and projectively equivariant quantizations and $s l(m+1, \mathbb{R})$-equivariant quantizations over $\mathbb{R}^{m}$ :

Proposition 1. If $Q_{M}$ is a natural projectively equivariant quantization and if $\nabla_{0}$ is the flat connection on $\mathbb{R}^{m}$, then $Q_{\mathbb{R}^{m}}\left(\nabla_{0}\right)$ is sl $(m+1, \mathbb{R})$-equivariant.

From now on to the end of this section, we will generalize the tools that were used by Duval, Lecomte and Ovsienko in [8], and generalized in [2] in order to obtain results of existence of such quantizations.

3.4. The affine quantization map. There exists a well-known bijection from symbols to differential operators over $\mathbb{R}^{m}$ : the so-called Standard ordering $Q_{A f f}$. If a symbol $T \in \mathcal{S}_{V_{1}, V_{2}}^{k}\left(\mathbb{R}^{m}\right)$ writes

$$
T(x, \xi)=\sum_{|\alpha|=k} C_{\alpha}(x) \xi^{\alpha},
$$

where $\alpha$ is a multi-index, $\xi \in \mathbb{R}^{m^{*}}$, and $C_{\alpha}(x) \in V_{1}^{*} \otimes V_{2}$, then one has

$$
Q_{A f f}(T)=\sum_{|\alpha|=k} C_{\alpha}(x) \circ\left(\frac{\partial}{\partial x}\right)^{\alpha} .
$$

From our point of view, $Q_{\text {Aff }}$ is an affinely equivariant quantization map. Indeed, it is easily seen that it exchanges the actions of the affine algebra (made of constant and linear vector fields) on the space of symbols and of differential operators.

Now, we can use formula (3) in order to express this quantization map in a coordinate-free manner : 
Proposition 2. If $h_{1}, \cdots, h_{k} \in \mathbb{R}^{m} \cong \mathfrak{g}_{-1}, A \in V_{1}^{*} \otimes V_{2}, t \in C^{\infty}\left(\mathbb{R}^{m}\right)$, and

$$
T(x)=t(x) A \otimes h_{1} \vee \cdots \vee h_{k},
$$

one has

$$
Q_{A f f}(T)=(-1)^{k} t(x) \circ A \circ L_{X^{h_{1}}} \circ \cdots \circ L_{X^{h_{k}}} .
$$

Proof. The proof is straightforward. Simply notice that we are dealing with constant vector fields.

3.5. The map $\gamma$. Using the affine quantization map, one can endow the space of symbols with a structure of representation of $\operatorname{Vect}\left(\mathbb{R}^{m}\right)$, isomorphic to $\mathcal{D}\left(\mathbb{R}^{m}\right)$. Explicitly, we set

$$
\mathcal{L}_{X} T=Q_{\text {Aff }}^{-1} \circ \mathcal{L}_{X} \circ Q_{\text {Aff }}(T),
$$

for every $T \in \mathcal{S}\left(\mathbb{R}^{m}\right)$ and $X \in \operatorname{Vect}\left(\mathbb{R}^{m}\right)$.

An equivariant quantization is then an $\operatorname{sl}(m+1, \mathbb{R})$-isomorphism from the representation $\left(\mathcal{S}\left(\mathbb{R}^{m}\right), L\right)$ to the representation $\left(\mathcal{S}\left(\mathbb{R}^{m}\right), \mathcal{L}\right)$.

In order to measure the difference between these representations the map

$$
\gamma: \mathfrak{g} \rightarrow \operatorname{gl}\left(\mathcal{S}\left(\mathbb{R}^{m}\right), \mathcal{S}\left(\mathbb{R}^{m}\right)\right): h \mapsto \gamma(h)=\mathcal{L}_{X^{h}}-L_{X^{h}}
$$

was introduced in [2. This map can be easily computed in coordinates, and we recall here its most important properties :

Proposition 3. The map $\gamma$ is a Chevalley-Eilenberg 1-cocycle and vanishes on $\mathfrak{g}_{-1} \oplus \mathfrak{g}_{0}$.

For every $h \in \mathfrak{g}_{1}$ and $k \in \mathbb{N}$, the restriction of $\gamma(h)$ to $\mathcal{S}^{k}\left(\mathbb{R}^{m}\right)$ has values in $\mathcal{S}^{k-1}\left(\mathbb{R}^{m}\right)$ and is a differential operator of order zero with constant coefficients.

For every $h, h^{\prime} \in \mathfrak{g}_{1}$, one has $\left[\gamma(h), \gamma\left(h^{\prime}\right)\right]=0$.

Remark : Since $\gamma(h)$ is a differential operator of order zero with constant coefficients, it is completely determined by its restriction to constant symbols.

For our purpose, it will be also interesting to obtain a coordinate free expression of $\gamma$. We have

Proposition 4. For every $h_{1}, \cdots, h_{k} \in \mathbb{R}^{m} \cong \mathfrak{g}_{-1}, A \in V_{1}^{*} \otimes V_{2}$ and $h \in \mathfrak{g}_{1} \cong \mathbb{R}^{m *}$ we have

$$
\begin{array}{r}
\gamma(h)\left(h_{1} \vee \cdots \vee h_{k} \otimes A\right)=\sum_{i=1}^{k} h_{1} \vee \cdots(i) \cdots \vee h_{k} \otimes\left(A \circ \rho_{1_{*}}\left(\left[h_{i}, h\right]\right)\right) \\
+\sum_{i=1}^{k} \sum_{j<i} h_{1} \vee \cdots(i, j) \cdots \vee h_{k} \vee\left[h_{j},\left[h_{i}, h\right]\right] \otimes A,
\end{array}
$$

where $\left[h_{i}, h\right]$ is in $g l(m, \mathbb{R})$ by the isomorphism (2).

Proof. By the very definition of $\gamma$, the expression

$$
Q_{A f f}\left(\gamma(h)\left(h_{1} \vee \cdots \vee h_{k} \otimes A\right)\right)
$$

is equal to

$$
\mathcal{L}_{X^{h}} \circ Q_{A f f}\left(h_{1} \vee \cdots \vee h_{k} \otimes A\right)-Q_{A f f}\left(L_{X^{h}}\left(h_{1} \vee \ldots \vee h_{k} \otimes A\right)\right) .
$$


This expression is a differential operator of order at most $k$. Its term of order $k$ vanishes : just apply the operator $\sigma$ to (5). Hence, we only have to sum up the terms of order less or equal to $k-1$ in the first term of expression (51). This latter term writes

$$
(-1)^{k}\left[L_{X^{h}} \circ A \circ L_{X^{h_{1}}} \circ \cdots \circ L_{X_{h_{k}}}-A \circ L_{X^{h_{1}}} \circ \cdots \circ L_{X^{h_{k}}} \circ L_{X^{h}}\right] .
$$

The first term is of order $k$ and $k+1$. The second term is

$$
\begin{aligned}
& -(-1)^{k} A \circ L_{X} \circ L_{X^{h_{1}}} \circ \cdots \circ L_{X^{h_{k}}} \\
& -(-1)^{k} \sum_{i=1}^{k} A \circ L_{X^{\left[h_{i}, h\right]}} \circ L_{X^{h_{1}}} \circ \cdots(i) \cdots \circ L_{X^{h_{k}}} \\
& -(-1)^{k} \sum_{i=1}^{k} \sum_{j<i} A \circ L_{X^{\left[h_{j}\left[h_{i}, h\right]\right]}} \circ L_{X^{h_{1}}} \circ \cdots(i, j) \cdots \circ L_{X^{h_{k}}},
\end{aligned}
$$

since $\left[h_{j}\left[h_{i}, h\right]\right]$ is a constant vector field.

The first summand is again of order $k$ and $k+1$. Now, in view of formula (11), the term of order $k-1$ in

$$
-(-1)^{k} \sum_{i=1}^{k} A \circ L_{X^{\left[h_{i}, h\right]}} \circ L_{X^{h_{1}}} \circ \cdots(i) \cdots \circ L_{X^{h_{k}}}
$$

is exactly

$$
(-1)^{k} \sum_{i=1}^{k} A \circ \rho_{1_{*}}\left(D X^{\left[h_{i}, h\right]}\right) \circ L_{X^{h_{1}}} \circ \cdots(i) \cdots \circ L_{X^{h_{k}}},
$$

and the result follows since $D X^{\left[h_{i}, h\right]}=-a d\left(\left[h_{i}, h\right]\right)$.

Now, the map $\gamma$ has an important invariance property :

Proposition 5. For every $a \in G L(m, \mathbb{R}), h \in \mathfrak{g}_{1}$ and every symbol $T$, one has

$$
\rho(a)(\gamma(h) T)=\gamma(A d(a) h)(\rho(a) T)
$$

Proof. The proof is a straightforward computation, using proposition 4 .

3.6. Casimir operators. In 8, 2, the construction of the quantization is based on the comparison of the spectra and of the eigenvectors of some (second order) Casimir operators. These operators are on the one hand the Casimir operator $C$ associated to the representation $\left(\mathcal{S}\left(\mathbb{R}^{m}\right), L\right)$ and on the other hand the Casimir operator $\mathcal{C}$ associated to the representation $\left(\mathcal{S}\left(\mathbb{R}^{m}\right), \mathcal{L}\right)$. We will briefly adapt the results of [2] in order to compute these operators.

From now on to the end of this section, we choose a basis $\left(e_{r}, h_{s}, \mathcal{E}, \epsilon^{t}\right)$ of $\operatorname{sl}(m+1, \mathbb{R})$, such that the bases $e_{i}$ and $\epsilon^{j}$ of $\mathfrak{g}_{-1}$ and $\mathfrak{g}_{1}$ are Killing-dual and $h_{j}$ is a basis of $\mathfrak{h}_{0}$. It was then proved in [2] that the dual basis writes $\left(\epsilon^{r}, h_{s}^{*}, \frac{1}{2 m} \mathcal{E}, e_{t}\right)$ and that moreover there holds

$$
\sum_{r=1}^{m}\left[e_{r}, \epsilon^{r}\right]=-\frac{1}{2} \mathcal{E} .
$$


We also set

$$
N=2 \sum_{i} \gamma\left(\epsilon^{i}\right) L_{X^{e_{i}}}
$$

The following result is then the direct generalization of the corresponding one in [2] :

Proposition 6. The Casimir operators are related by

$$
\mathcal{C}=C+N
$$

The next step is to analyse the eigenvalue problem for the operator $C$. To this aim, we have to fix some more notation : as a representation of $\mathfrak{h}_{0} \cong \operatorname{sl}(m, \mathbb{R}), S_{V_{1}, V_{2}}^{k}=S^{k} \mathbb{R}^{m} \otimes V_{1}^{*} \otimes V_{2}$ decomposes as a sum of irreducible representations

$$
S_{V_{1}, V_{2}}^{k}=\oplus_{s=1}^{n_{k}} I_{k, s}
$$

For each irreducible representation $I_{k, s}$ we denote by $E_{k, s}$ the corresponding space of sections, that is

$$
E_{k, s}=C^{\infty}\left(\mathbb{R}^{m}, I_{k, s}\right) .
$$

Furthermore, in $\operatorname{sl}(m, \mathbb{C})$, we consider the usual Cartan subalgebra $\mathfrak{C}$ made of diagonal and traceless matrices. We consider the elements of $\mathfrak{C}^{*}$ defined by

$$
\delta_{i}\left(\operatorname{diag}\left(a_{1}, \cdots, a_{m}\right)\right)=a_{i} .
$$

It is known that a simple root system is given by $\left\{\delta_{i}-\delta_{i+1},(i=1, \ldots, m-\right.$ $1)\}$. The Weyl vector is defined as half the sum of the positive roots and is given by

$$
\rho_{S}=\sum_{i}(m-i) \delta_{i}
$$

The Killing form of $\operatorname{sl}(m, \mathbb{C})$ is the extension of the Killing form of $\operatorname{sl}(m, \mathbb{R})$ and induces a scalar product $($,$) on the real vector space spanned by the$ roots.

Now for each irreducible representation $I_{k, s}$ of $s l(m, \mathbb{R})$, the complexified representation $I_{k, s} \otimes \mathbb{C}$ of $s l(m, \mathbb{C})$ is also irreducible and we denote by $\mu_{I_{k, s}}$ its highest weight.

In view of the definitions of the representations $V_{1}$ and $V_{2}$, there exist real numbers $a_{1}$ and $a_{2}$ such that

$$
\left.\rho_{*}(I d)\right|_{V_{i}}=a_{i} I d .
$$

In order to be consistent we respect to the definition of the shift value in [2, 8], we define the shift of the pair $\left(V_{1}, V_{2}\right)$ by

$$
\delta=\frac{1}{m}\left(a_{1}-a_{2}\right) .
$$

We can now state the main result concerning the operator $C$ : 
Theorem 7. The space of symbols $\mathcal{S}\left(\mathbb{R}^{m}\right)$ is the direct sum of eigenspaces of $C$. Precisely, for every $k \in \mathbb{N}$, the restriction of $C$ to $E_{k, s}$ is equal to $\alpha_{k, s} I d_{E_{k, s}}$ where

$$
\alpha_{k, s}=\frac{1}{2 m}(m \delta-k)(m(\delta-1)-k)+\frac{m}{m+1}\left(\mu_{I_{k, s}}, \mu_{I_{k, s}}+2 \rho_{S}\right) .
$$

Proof. With our choice of dual bases, the operator $C$ writes

$$
\sum_{i}\left(L_{X^{\epsilon^{i}}} \circ L_{X^{e_{i}}}+L_{X^{e_{i}}} \circ L_{X^{\epsilon^{i}}}\right)+\frac{1}{2 m}\left(L_{X^{\mathcal{E}}}\right)^{2}+\sum_{j} L_{X^{h_{j}}} \circ L_{X^{h_{j}^{*}}},
$$

that is, using relation (7),

$$
2 \sum_{i}\left(L_{X^{\epsilon^{i}}} \circ L_{X^{e_{i}}}\right)-\frac{1}{2} L_{X^{\mathcal{E}}}+\frac{1}{2 m}\left(L_{X^{\mathcal{E}}}\right)^{2}+\sum_{j} L_{X^{h_{j}}} \circ L_{X^{h_{j}^{*}}} .
$$

Since $C$ commutes with $L_{X^{h}}$ for all $h \in \mathfrak{g}_{-1} \cong \mathbb{R}^{m}$ it must have constant coefficients. Hence, we only take such terms into account. We then use the expression of the Lie derivative (11), and the expression of the vector fields $X^{h}$, and get

$$
C=-\frac{1}{2} \rho_{*}(a d(\mathcal{E}))+\frac{1}{2 m}\left(\rho_{*}(a d(\mathcal{E}))\right)^{2}+\sum_{j} \rho_{*}\left(\operatorname{ad}\left(h_{j}\right)\right) \circ \rho_{*}\left(a d\left(h_{j}\right)\right),
$$

where $a d$ is the adjoint representation of $\mathfrak{g}_{0}$ on $\mathfrak{g}_{-1}$. The terms involving the Euler element can be easily computed since $\left.a d(\mathcal{E})\right|_{\mathfrak{g}_{-1}}=-I d$. Now, the restriction of the Killing form of $\operatorname{sl}(m+1, \mathbb{R})$ to the subalgebra $\operatorname{sl}(m, \mathbb{R})$ is $\frac{m+1}{m}$ times the Killing form of $s l(m, \mathbb{R})$, so that the bases $\left(h_{j}\right)$ and $\left(\frac{m+1}{m} h_{j}^{*}\right)$ are dual with respect to the latter. Then

$$
\sum_{j} \rho_{*}\left(a d\left(h_{j}\right)\right) \circ \rho_{*}\left(a d\left(h_{j}\right)\right)=\frac{m}{m+1} C^{\prime},
$$

where $C^{\prime}$ is the Casimir operator of $\operatorname{sl}(m, \mathbb{R})$ acting on $I_{k, s}$ or the Casimir operator of $\operatorname{sl}(m, \mathbb{C})$ acting on $I_{k, s} \otimes \mathbb{C}$. It is well known (see for instance 13 , p. 122]) that this operator equals

$$
\left(\mu_{I_{k, s}}, \mu_{I_{k, s}}+2 \rho_{S}\right)
$$

times the identity of $I_{k, s}$.

3.7. Tree-like subspaces and critical situations. In order to analyse the spectrum of the operator $\mathcal{C}$ we introduce, as in 2, 8, the treelike susbspaces associated to an irreducible representation $I_{k, s} \subset S_{V_{1}, V_{2}}^{k}$ : we set

$$
\mathcal{T}_{\gamma}\left(I_{k, s}\right)=\bigoplus_{l \in \mathbb{N}} \mathcal{T}_{\gamma}^{l}\left(I_{k, s}\right)
$$

where $\mathcal{T}_{\gamma}^{0}\left(I_{k, s}\right)=I_{k, s}$ and $\mathcal{T}_{\gamma}^{l+1}\left(I_{k, s}\right)=\gamma\left(\mathfrak{g}_{1}\right)\left(\mathcal{T}_{\gamma}^{l}\left(I_{k, s}\right)\right)$, for all $l \in \mathbb{N}$. The spaces $\mathcal{T}_{\gamma}^{l}\left(E_{k, s}\right)$ are defined in the same way, so that

$$
\mathcal{T}_{\gamma}^{l}\left(E_{k, s}\right)=C^{\infty}\left(\mathbb{R}^{m}, \mathcal{T}_{\gamma}^{l}\left(I_{k, s}\right)\right)
$$


for all $l \in \mathbb{N}$.

The spaces $\mathcal{T}_{\gamma}\left(E_{k, s}\right)$ have a nice property :

Proposition 8. The space $\mathcal{T}_{\gamma}\left(E_{k, s}\right)$ is stable under the actions of $L_{X^{h}}$ and $\mathcal{L}_{X^{h}}$, for every $h \in \operatorname{sl}(m+1, \mathbb{R})$.

Proof. Proposition [5 allows to prove by induction that $\mathcal{T}_{\gamma}\left(E_{k, s}\right)$ is stable under the action of $\rho_{*}(A)$ for all $A \in g l(m, \mathbb{R})$. It is then clearly stable under the Lie derivative $L_{X^{h}}$ for all $h \in \operatorname{sl}(m+1, \mathbb{R})$ because of the expression (11) of $L_{X^{h}}$. The result then follows since $\mathcal{L}_{X^{h}}=L_{X^{h}}+\gamma(h)$.

The following definition is a direct generalization of the ones of [2, 8] :

Definition 1. An ordered pair of representations $\left(V_{1}, V_{2}\right)$ is critical if there exists $k, s$ such that the eigenvalue $\alpha_{k, s}$ belongs to the spectrum of the restriction of $C$ to $\bigoplus_{l \geq 1} \mathcal{T}_{\gamma}^{l}\left(E_{k, s}\right)$.

3.8. Construction of the quantization. The result is the following :

Theorem 9. If $\left(V_{1}, V_{2}\right)$ is not critical, then there exists a projectively equivariant quantization from $\mathcal{S}_{V_{1}, V_{2}}\left(\mathbb{R}^{m}\right)$ to $\mathcal{D}\left(\mathcal{V}_{1}\left(\mathbb{R}^{m}\right), \mathcal{V}_{2}\left(\mathbb{R}^{m}\right)\right)$.

Proof. The proof is as in [2] and [8]. We give here the main ideas for the sake of completeness.

First remark that for every $T \in E_{k, s}$ there exists a unique eigenvector $\hat{T}$ of $\mathcal{C}$ with eigenvalue $\alpha_{k, s}$ such that

$$
\left\{\begin{array}{l}
\hat{T}=T_{k}+T_{k-1}+\cdots+T_{0}, \quad T_{k}=T \\
T_{l} \in \mathcal{T}_{\gamma}^{k-l}\left(E_{k, s}\right) \quad \text { for all } l \leq k-1
\end{array}\right.
$$

Indeed, these conditions write

$$
\left\{\begin{array}{l}
C(T)=\alpha_{k, s} T \\
\left.\left(C-\alpha_{k, s} \mathrm{Id}\right)\right) T_{k-l}=-N\left(T_{k-l+1}\right) \quad \forall l \in\{1, \cdots, k\} \\
T_{k-l} \in \mathcal{T}_{\gamma}^{l}\left(E_{k, s}\right)
\end{array}\right.
$$

The first condition is satisfied since $T$ belongs to $E_{k, s}$. For the second and third ones, remark that if $T_{k-l+1}$ is in $\mathcal{T}_{\gamma}^{l-1}\left(E_{k, s}\right)$ then $N\left(T_{k-l+1}\right)$ belongs to $\mathcal{T}_{\gamma}^{l}\left(E_{k, s}\right)$, by proposition 8 Now, $\mathcal{T}_{\gamma}^{l}\left(E_{k, s}\right)$ decomposes as a direct sum of eigenspaces of $C$, as theorem 7 shows. The restriction of the operator $C-\alpha_{k, s}$ Id to each of these eigenspaces is a non-vanishing scalar multiple of the identity, hence the existence and uniqueness of $T_{k-l}$.

Now, define the quantization $Q$ by

$$
\left.Q\right|_{E_{k, s}}(T)=\hat{T}
$$

It is clearly a bijection.

It also fulfils

$$
Q \circ L_{X^{h}}=\mathcal{L}_{X^{h}} \circ Q \quad \forall h \in \operatorname{sl}(m+1, \mathbb{R}) .
$$

Indeed, for all $T \in E_{k, s}, Q\left(L_{X^{h}} T\right)$ and $\mathcal{L}_{X^{h}}(Q(T))$ share the following properties 
- They are eigenvectors of $\mathcal{C}$ of eigenvalue $\alpha_{k, s}$ because on the one hand $\mathcal{C}$ commutes with $\mathcal{L}_{X^{h}}$ for all $h$ and on the other hand $L_{X^{h}} T$ belongs to $E_{k, s}$ by proposition 8

- their term of degree $k$ is exactly $L_{X^{h}} T$,

- they belong to $\mathcal{T}_{\gamma}\left(E_{k, s}\right)$ by proposition 8

The first part of the proof shows that they have to coincide.

3.9. A technical result. The following proposition will be fundamental for our purpose :

Proposition 10. The relation

$$
[\gamma(h), C]=2 \sum_{i} \gamma\left(\epsilon^{i}\right) \rho_{*}\left(\left[h, e_{i}\right]\right)
$$

holds for all $h \in \mathfrak{g}_{1}$.

Proof. As a Casimir operator commutes with the corresponding representation, we have :

$$
\begin{aligned}
& {\left[\mathcal{L}_{X^{h}}, \mathcal{C}\right]=0,} \\
& {\left[L_{X^{h}}, C\right]=0 .}
\end{aligned}
$$

These equations lead to

$$
\left[L_{X^{h}}, N\right]+[\gamma(h), C]+[\gamma(h), N]=0 .
$$

It is easily seen using proposition 3 that $[\gamma(h), N]$ vanishes. Moreover, we have

$$
\left[L_{X^{h}}, N\right]=2 \sum_{i}\left(L_{X^{h}} \gamma\left(\epsilon^{i}\right) L_{X^{e_{i}}}-\gamma\left(\epsilon^{i}\right) L_{X^{e_{i}}} L_{X^{h}}\right) .
$$

The terms of order greater than zero in this expression must vanish because $[\gamma(h), C]$ has order zero. Hence we only collect the terms of order zero.

Using formula (11), we see that the first term is of order greater or equal to 1 . The second term writes

$$
-2 \gamma\left(\epsilon^{i}\right) \sum_{i}\left(L_{X^{h}} L_{X^{e_{i}}}+L_{X^{\left[e_{i}, h\right]}}\right)
$$

The terms of order zero in this expression are

$$
2 \sum_{i} \gamma\left(\epsilon^{i}\right) \rho_{*}\left(D X^{\left[e_{i}, h\right]}\right)
$$

hence the result.

\section{TOOLS OF THE CURVED CASE}

Here we will adapt the tools presented in section 3 to the curved situation. 
4.1. Projective structures and Cartan projective connections. These tools were presented in detail in [18, Section 3]. We give here the most important ones for this paper to be self-contained.

We consider the group $G=P G L(m+1, \mathbb{R})$ described in section 3.2 . We denote by $H$ the subgroup associated to the subalgebra $\mathfrak{g}_{0} \oplus \mathfrak{g}_{1}$, that is

$$
H=\left\{\left(\begin{array}{cc}
A & 0 \\
\xi & a
\end{array}\right): A \in G L(m, \mathbb{R}), \xi \in \mathbb{R}^{m *}, a \neq 0\right\} / \mathbb{R}_{0} \mathrm{Id}
$$

The group $H$ is the semi-direct product $G_{0} \rtimes G_{1}$, where $G_{0}$ is isomorphic to $G L(m, \mathbb{R})$ and $G_{1}$ is isomorphic to $\mathbb{R}^{m *}$.

Then there is a projection

$$
\pi: H \rightarrow G L(m, \mathbb{R}):\left[\left(\begin{array}{cc}
A & 0 \\
\xi & a
\end{array}\right)\right] \mapsto \frac{A}{a}
$$

It is well-known that $H$ can be seen as a subgroup of the group of 2-jets $G_{m}^{2}$, an explicit formula is given in [14, 18.

A Projective structure on $M$ is then a reduction of the second order jetbundle $P^{2} M$ to the group $H$.

The following result (14, P. 147]) is the starting point of our method :

Proposition 11 (Kobayashi-Nagano). There is a natural one to one correspondence between the projective equivalence classes of torsion-free linear connections on $M$ and the projective structures on $M$.

We now recall the definition of a projective Cartan connection :

Definition 2. Let $P \rightarrow M$ be a principal $H$-bundle. A projective Cartan connection on $P$ is an $s l(m+1, \mathbb{R})$ - valued 1 -form $\omega$ such that

- There holds $R_{a}^{*} \omega=A d\left(a^{-1}\right) \omega, \quad \forall a \in H$,

- One has $\omega\left(k^{*}\right)=k \quad \forall k \in h=\mathfrak{g}_{0} \oplus \mathfrak{g}_{1}$,

- For all $u \in P, \omega_{u}: T_{u} P \rightarrow s l(m+1, \mathbb{R})$ is a linear bijection.

In general, if $\omega$ is a Cartan connection defined on a $H$-principal bundle $P$, then its curvature $\Omega$ is defined as usual by

$$
\Omega=d \omega+\frac{1}{2}[\omega, \omega]
$$

The notion of Normal Cartan connection is defined by natural conditions imposed on the components of the curvature.

Now, the following result ([14, p. 135]) gives the relationship between projective structures and Cartan connections :

Proposition 12. A unique normal Cartan projective connection is associated to every projective structure $P$. This association is natural.

The connection associated to a projective structure $P$ is called the normal projective connection of the projective structure. 
4.2. Lift of equivariant functions. As we continue, we will need to know the relationship between equivariant functions on $P^{1} M$ and equivariant functions on $P$. The following results were already quoted in [6, p. 47].

If $(V, \rho)$ is a representation of $G L(m, \mathbb{R})$, then we define a representation $\left(V, \rho^{\prime}\right)$ of $H$ by

$$
\rho^{\prime}: H \rightarrow G L(V):\left[\left(\begin{array}{cc}
A & 0 \\
\xi & a
\end{array}\right)\right] \mapsto \rho \circ \pi\left(\left[\left(\begin{array}{cc}
A & 0 \\
\xi & a
\end{array}\right)\right]\right)=\rho\left(\frac{A}{a}\right)
$$

for every $A \in G L(m, \mathbb{R}), \xi \in \mathbb{R}^{m *}, a \neq 0$.

Now, using the representation $\rho^{\prime}$, we can give the relationship between equivariant functions on $P^{1} M$ and equivariant functions on $P$ : If $P$ is a projective structure on $M$, the natural projection $P^{2} M \rightarrow P^{1} M$ induces a projection $p: P \rightarrow P^{1} M$ and we have a well-know result:

Proposition 13. If $(V, \rho)$ is a representation of $G L(m, \mathbb{R})$, then the map

$$
p^{*}: C^{\infty}\left(P^{1} M, V\right) \rightarrow C^{\infty}(P, V): f \mapsto f \circ p
$$

defines a bijection from $C^{\infty}\left(P^{1} M, V\right)_{\mathrm{GL}(m, \mathbb{R})}$ to $C^{\infty}(P, V)_{H}$.

Now, since $\mathbb{R}^{m}$ and $\mathbb{R}^{m *}$ are natural representations of $G L(m, \mathbb{R})$, they become representations of $H$ and we can state an important property of the invariant differentiation :

Proposition 14. If $f$ belongs to $C^{\infty}(P, V)_{G_{0}}$ then $\nabla^{\omega} f \in C^{\infty}\left(P, \mathbb{R}^{m *} \otimes\right.$ $V)_{G_{0}}$.

Proof. The result is a direct consequence of the Ad-invariance of the Cartan connection $\omega$.

The main point that we will discuss in the next sections is that this result is not true in general for $H$-equivariant functions : for an $H$-equivariant function $f$, the function $\nabla^{\omega} f$ is in general not $G_{1}$-equivariant.

As we continue, we will use the representation $\rho_{*}^{\prime}$ of the Lie algebra of $H$ on $V$. If we recall that this algebra is isomorphic to $g l(m, \mathbb{R}) \oplus \mathbb{R}^{m *}$ then we have

$$
\rho_{*}^{\prime}(A, \xi)=\rho_{*}(A), \quad \forall A \in g l(m, \mathbb{R}), \xi \in \mathbb{R}^{m *} .
$$

In our computations, we will make use of the infinitesimal version of the equivariance relation : If $f \in C^{\infty}(P, V)_{H}$ then one has

$$
L_{h^{*}} f(u)+\rho_{*}^{\prime}(h) f(u)=0, \quad \forall h \in g l(m, \mathbb{R}) \oplus \mathbb{R}^{m *} \subset \operatorname{sl}(m+1, \mathbb{R}), \forall u \in P .
$$

4.3. The curved affine quantization map. The construction of the curved analog of the affine quantization map is based on the concept of invariant differentiation developed in 6, 7]. Let us recall the definition :

Definition 3. Let $(V, \rho)$ be a representation of $H$. If $f \in C^{\infty}(P, V)$, then the invariant differential of $f$ with respect to $\omega$ is the function $\nabla^{\omega} f \in C^{\infty}\left(P, \mathbb{R}^{m *} \otimes V\right)$ defined by

$$
\nabla^{\omega} f(u)(X)=L_{\omega^{-1}(X)} f(u) \quad \forall u \in P, \quad \forall X \in \mathbb{R}^{m} .
$$


We will also use an iterated and symmetrized version of the invariant differentiation

Definition 4. If $f \in C^{\infty}(P, V)$ then $\left(\nabla^{\omega}\right)^{k} f \in C^{\infty}\left(P, S^{k} \mathbb{R}^{m *} \otimes V\right)$ is defined by

$$
\left(\nabla^{\omega}\right)^{k} f(u)\left(X_{1}, \ldots, X_{k}\right)=\frac{1}{k !} \sum_{\nu} L_{\omega^{-1}\left(X_{\nu_{1}}\right)} \circ \ldots \circ L_{\omega^{-1}\left(X_{\nu_{k}}\right)} f(u)
$$

for $X_{1}, \ldots, X_{k} \in \mathbb{R}^{m}$.

Using this iterated version, we can transform a symbol $T \in C^{\infty}\left(P, S_{V_{1}, V_{2}}^{k}\right)$ into a differential operator $Q_{\omega}(T)$ acting on functions $f \in C^{\infty}\left(P, V_{1}\right)$ by setting

$$
Q_{\omega}(T)(f)=\left\langle T,\left(\nabla^{\omega}\right)^{k} f\right\rangle .
$$

Explicitly, when the symbol $T$ writes $t A \otimes h_{1} \vee \cdots \vee h_{k}$ for $t \in C^{\infty}(P)$, $A \in V_{1}^{*} \otimes V_{2}$ and $h_{1}, \cdots, h_{k} \in \mathbb{R}^{m} \cong \mathfrak{g}_{-1}$ then one has

$$
Q_{\omega}(T) f=\frac{1}{k !} \sum_{\nu} t A \circ L_{\omega^{-1}\left(h_{\nu_{1}}\right)} \circ \cdots \circ L_{\omega^{-1}\left(h_{\nu_{k}}\right)} f,
$$

where $\nu$ runs over all permutations of the indices $\{1, \cdots, k\}$ and $t$ is considered as a multiplication operator.

Remark 1. If $T \in C^{\infty}\left(P, S_{V_{1}, V_{2}}^{k}\right)$ is $H$-equivariant, the differential operator $Q_{\omega}(T)$ does not transform $H$-equivariant functions into $H$-equivariant functions. Indeed, when $f$ is $H$-equivariant, the function $\left(\nabla^{\omega}\right)^{k} f$ is only $G_{0}$-equivariant. Hence the function $Q_{\omega}(T) f$ does not correspond to a section of $\mathcal{V}_{2}(M)$. As we continue, we will show that one can modify the symbol $T$ by lower degree correcting terms in order to solve this problem.

4.4. Measuring the default of equivariance. Throughout this section, $T$ will denote an element of $C^{\infty}\left(P, S_{V_{1}, V_{2}}^{k}\right)_{G_{0}}$ and $f \in C^{\infty}\left(P, V_{1}\right)_{G_{0}}$ (remark that this ensures that $Q_{\omega}(T)(f)$ is in $\left.C^{\infty}\left(P, V_{2}\right)_{G_{0}}\right)$. Now, in order to analyse the invariance of functions, we have this first easy result

Proposition 15. If $(V, \rho)$ is a representation of $G_{0}$ and becomes a representation of $H$ as stated in section 4.2, then a function $v \in C^{\infty}(P, V)$ is $H$-equivariant iff

$$
\left\{\begin{array}{l}
v \text { is } G_{0}-\text { equivariant } \\
\text { One has } L_{h^{*}} v=0 \text { for every } h \text { in } \mathfrak{g}_{1}
\end{array}\right.
$$

Proof. Of course, $H$-equivariance is equivalent to $G_{0}-$ and $G_{1}$-equivariance. Now, $G_{1}$-equivariance is equivalent to $\mathfrak{g}_{1}$-equivariance since $G_{1}$ is a vector space. The result follows since $G_{1}$ acts trivially on $V$.

Since basically, our tools preserve the $G_{0}$-equivariance, we are mostly interested in the $\mathfrak{g}_{1}$-equivariance. The following result is the keystone of our method : 
Proposition 16. The relation

$$
L_{h^{*}} Q_{\omega}(T)(f)-Q_{\omega}(T)\left(L_{h^{*}} f\right)=Q_{\omega}\left(\left(L_{h^{*}}+\gamma(h)\right) T\right)(f)
$$

holds for all $f \in C^{\infty}\left(P, V_{1}\right)_{G_{0}}, h \in \mathfrak{g}_{1}$, and $T \in C^{\infty}\left(P, S_{V_{1}, V_{2}}^{k}\right)$.

Proof. The relation that we have to prove writes

$$
\left\langle T, L_{h^{*}}\left(\nabla^{\omega}\right)^{k} f-\left(\nabla^{\omega}\right)^{k} L_{h^{*}} f\right\rangle=\left\langle\gamma(h) T,\left(\nabla^{\omega}\right)^{k-1} f\right\rangle .
$$

Since both sides are $C^{\infty}(P)$-linear in $T$, it is sufficient to check this relation for a constant symbol $T$ that has the form $X^{k} \otimes A$, where $X \in \mathfrak{g}_{-1}$ and $A \in V_{1}^{*} \otimes V_{2}$. Then the left-hand side writes

$$
A\left(L_{h *} L_{\omega^{-1}(X)} \ldots L_{\omega^{-1}(X)} f-L_{\omega^{-1}(X)} \ldots L_{\omega^{-1}(X)} L_{h^{*}} f\right)
$$

and is equal to

$$
\begin{aligned}
& A\left(\sum_{j=1}^{k} L_{\omega^{-1}(X)} \ldots L_{[h, X]^{*}}^{(j)} \ldots L_{\omega^{-1}(X)} f\right) \\
& =A\left(\sum_{j=1}^{k-1} \sum_{i>j} L_{\omega^{-1}(X)} \cdot{ }^{(j)} \cdot L_{\omega^{-1}([[h, X], X])} \ldots L_{\omega^{-1}(X)} f\right) \\
& -A\left(\sum_{i=1}^{k} L_{\omega^{-1}(X)}{ }^{(i)} \cdot L_{\omega^{-1}(X)} \rho_{1_{*}}([h, X]) f\right) .
\end{aligned}
$$

Moreover, one has

$$
[[h, X], X]=-2\langle h, X\rangle X
$$

and the result follows by proposition 4

4.5. Curved Casimir operators. The parallelism between the flat and curved situation suggests to define an analog of the operator $\mathcal{C}$.

We first define the analog of $N$ by setting

$$
N^{\omega}=-2 \sum_{i} \gamma\left(\epsilon^{i}\right) L_{\omega^{-1}\left(e_{i}\right)} .
$$

Then we can define the operators $C^{\omega}$ and $\mathcal{C}^{\omega}$ by their restrictions to the spaces $C^{\infty}\left(P, I_{k, s}\right)$ : for all $T \in C^{\infty}\left(P, I_{k, s}\right)$, we set

$$
\left\{\begin{array}{l}
C^{\omega}(T)=\alpha_{k, s} T \\
\mathcal{C}^{\omega}(T)=C^{\omega}(T)+N^{\omega}(T)
\end{array}\right.
$$

where $\alpha_{k, s}$ is the eigenvalue of $C$ on $E_{k, s}=C^{\infty}\left(\mathbb{R}^{m}, I_{k, s}\right)$.

The operator $\mathcal{C}^{\omega}$ has the following property

Proposition 17. For every $h \in \mathfrak{g}_{1}$, one has

$$
\left[L_{h^{*}}+\gamma(h), \mathcal{C}^{\omega}\right]=0
$$

on $C^{\infty}\left(P, S_{V_{1}, V_{2}}^{k}\right)_{G_{0}}$.

Proof. First we have

$$
\left[L_{h^{*}}+\gamma(h), C^{\omega}+N^{\omega}\right]=\left[L_{h^{*}}, C^{\omega}\right]+\left[L_{h^{*}}, N^{\omega}\right]+\left[\gamma(h), C^{\omega}\right]+\left[\gamma(h), N^{\omega}\right] .
$$

Next $\left[L_{h^{*}}, C^{\omega}\right]=0$ because $L_{h^{*}}$ stabilizes each eigenspace $C^{\infty}\left(P, I_{k, s}\right)$ of $C^{\omega}$. In the same way, we have $\left[\gamma(h), N^{\omega}\right]=0$ since

- by proposition $3\left[\gamma(h), \gamma\left(\epsilon^{i}\right)\right]=0$, 
- $\left[\gamma(h), L_{\omega^{-1}\left(e_{i}\right)}\right]=0$ because $\gamma(h)$ only acts on the target space $S_{V_{1}, V_{2}}^{k}$. Eventually, we have

$$
\begin{aligned}
{\left[L_{h^{*}}, N^{\omega}\right] } & =-2 \sum_{i} \gamma\left(\epsilon^{i}\right)\left[L_{h^{*}}, L_{\omega^{-1}\left(e_{i}\right)}\right] \\
& =-2 \sum_{i} \gamma\left(\epsilon^{i}\right) L_{\left[h, e_{i}\right]^{*}} \\
& =2 \sum_{i} \gamma\left(\epsilon^{i}\right) \rho_{*}\left(\left[h, e_{i}\right]\right)
\end{aligned}
$$

by the $G_{0}$-equivariance. We conclude by proposition 10 .

For the operator $N^{\omega}$, we have the following result :

Proposition 18. The operator $N^{\omega}$ preserves the $G_{0}$-equivariance of functions.

Proof. This property is a consequence of the proposition 5 and of the fact that the invariant differentiation preserves the $G_{0}$-equivariance. One has successively, for all $f \in C^{\infty}\left(P, S_{V_{1}, V_{2}}^{k}\right), u \in P$ and $g \in G_{0}$ :

$$
\begin{aligned}
\left(N^{\omega}(f)\right)(u g) & =\sum_{i}\left(\gamma\left(\epsilon^{i}\right) L_{\omega^{-1}\left(e_{i}\right)} f\right)(u g) \\
& =\sum_{i} \gamma\left(\epsilon^{i}\right)\left(\nabla^{\omega} f\right)(u g)\left(e_{i}\right) \\
& =\sum_{i} \gamma\left(\epsilon^{i}\right) \rho\left(g^{-1}\right)\left(\left(\nabla^{\omega} f\right)(u)\left(A d(g) e_{i}\right)\right) \\
& =\sum_{i} \rho\left(g^{-1}\right)\left(\gamma\left(A d(g) \epsilon^{i}\right)\left(\nabla^{\omega} f\right)(u)\left(A d(g) e_{i}\right)\right) \\
& =\rho\left(g^{-1}\right) \sum_{i} \gamma\left(\epsilon^{i}\right) L_{\omega^{-1}\left(e_{i}\right)} f(u),
\end{aligned}
$$

hence the result.

\section{Construction of the quantization}

The construction of the quantization is based on the eigenvalue problem for the operator $\mathcal{C}^{\omega}$.

First remark that the construction of section 3.8 is still valid in the curved case.

Theorem 19. If the pair $\left(V_{1}, V_{2}\right)$ is not critical, for every $T$ in $C^{\infty}\left(P, I_{k, s}\right)$, there exists a unique function $\hat{T}$ in $C^{\infty}\left(P, \mathcal{T}_{\gamma}\left(I_{k, s}\right)\right)$ such that

$$
\begin{cases}\hat{T} & =T_{k}+\cdots+T_{0}, \quad T_{k}=T \\ \mathcal{C}^{\omega}(\hat{T}) & =\alpha_{k, s} \hat{T} .\end{cases}
$$

Moreover, if $T$ is $G_{0}$-invariant, then $\hat{T}$ is $G_{0}$-invariant.

Proof. The function $\hat{T}$ exists and is unique. Simply notice that the conditions in (17) are nothing but the ones in (11) with $C$ replaced by $C^{\omega}$ and $N$ replaced by $N^{\omega}$. Now, the main point in order to solve (11) was that $N$ maps $\mathcal{T}_{\gamma}^{l-1}\left(E_{k, s}\right)$ into $\mathcal{T}_{\gamma}^{l}\left(E_{k, s}\right)$. This latter fact is actually a property of $\gamma$ and therefore we have

$$
N^{\omega}\left(C^{\infty}\left(P, \mathcal{T}_{\gamma}^{l-1}\left(I_{k, s}\right)\right)\right) \subset C^{\infty}\left(P, \mathcal{T}_{\gamma}^{l}\left(I_{k, s}\right)\right) .
$$

Suppose in addition that $T$ is $G_{0}$-invariant, then $\hat{T}$ is $G_{0}$-invariant. Indeed, $\hat{T}$ is obtained from $T$ by applying successively the operators $N^{\omega}$ and the projectors from $\mathcal{T}_{\gamma}^{l}\left(I_{k, s}\right)$ ) onto its irreducible components, and these operations preserve the $G_{0}$-equivariance of functions (see prop 18). 
This result allows to define the main ingredient in order to define the quantization.

Definition 5. Suppose that the pair $\left(V_{1}, V_{2}\right)$ is not critical. Then the map

$$
Q: C^{\infty}\left(P, S_{V_{1}, V_{2}}\right) \rightarrow C^{\infty}\left(P, S_{V_{1}, V_{2}}\right)
$$

is the linear extension of the association $T \mapsto \hat{T}$.

The map $Q$ has the following nice property :

Proposition 20. There holds

$$
\left(L_{h^{*}}+\gamma(h)\right) Q(T)=Q\left(L_{h^{*}} T\right)
$$

for every $h \in \mathfrak{g}_{1}$ and every $T \in C^{\infty}\left(P, S_{V_{1}, V_{2}}\right)_{G_{0}}$.

Proof. The proof is just an adaptation of the one of theorem 9, It is sufficient to check the property for $T \in C^{\infty}\left(P, I_{k, s}\right)_{G_{0}}$ (for all $k$ and $s$ ). For such a $T$, the function $Q\left(L_{h^{*}} T\right)$ is defined by (17) : it is the unique eigenvector of $\mathcal{C}^{\omega}$ in $C^{\infty}\left(P, \mathcal{T}_{\gamma}\left(I_{k, s}\right)\right)$ corresponding to the eigenvalue $\alpha_{k, s}$ and with highest degree term $L_{h^{*}} T$. The left hand side of equation (18) has $L_{h^{*}} T$ as highest degree term since $\gamma(h)$ lowers the degree of its arguments. It belongs clearly to $C^{\infty}\left(P, \mathcal{T}_{\gamma}\left(I_{k, s}\right)\right)$. Finally, since $Q(T)$ is $G_{0}$-invariant, proposition 17 implies that $\left(L_{h^{*}}+\gamma(h)\right) Q(T)$ is an eigenvector of $\mathcal{C}^{\omega}$ with eigenvalue $\alpha_{k, s}$.

These rather technical results allow to state the main theorem.

Theorem 21. If the pair $\left(V_{1}, V_{2}\right)$ is not critical, then the formula

$$
Q_{M}:(\nabla, T) \mapsto Q_{M}(\nabla, T)(f)=\left(p^{*}\right)^{-1}\left[Q_{\omega}\left(Q\left(p^{*} T\right)\right)\left(p^{*} f\right)\right],
$$

defines a natural and projectively equivariant quantization.

Proof. First, the formula makes sense : the function $Q_{\omega}\left(Q\left(p^{*} T\right)\right)\left(p^{*} f\right)$ is $H$-equivariant. The $G_{0}$-equivariance follows from theorem 19 and remark 1 . The $\mathfrak{g}_{1}$-equivariance follows from the relations

$$
\begin{aligned}
L_{h^{*}}\left[Q_{\omega}\left(Q\left(p^{*} T\right)\right)\left(p^{*} f\right)\right] & =\mathcal{L}_{h^{*}}\left[Q_{\omega}\left(Q\left(p^{*} T\right)\right)\right]\left(p^{*} f\right) \\
& =Q_{\omega}\left[\left(L_{h^{*}}+\gamma(h)\right)\left(Q\left(p^{*} T\right)\right)\right]\left(p^{*} f\right) \\
& =Q_{\omega}\left[Q\left(L_{h^{*}}\left(p^{*} T\right)\right)\right]\left(p^{*} f\right) .
\end{aligned}
$$

Next, the principal symbol of $Q_{M}(\nabla, T)(f)$ is exactly $T$. Simply notice that the leading term of $Q\left(p^{*} T\right)$ is $p^{*} T$, then use the results of [7, p. 47].

Hence, $Q_{M}(\nabla)$ is a quantization. It is also projectively equivariant by the very definition of $\omega$.

Eventually, the naturality of the so defined equivariant quantizations is a consequence of the naturality of all the tools used to derive the formula.

\section{ACKNowledgements}

F. Radoux thanks the Belgian FRIA for his Research Fellowship. 


\section{REFERENCES}

[1] F. Boniver, S. Hansoul, P. Mathonet, and N. Poncin. Equivariant symbol calculus for differential operators acting on forms. Lett. Math. Phys., 62(3):219-232, 2002.

[2] F. Boniver and P. Mathonet. Ifft-equivariant quantizations. To appear in J. Geom. Phys., math.RT/0206213, 2005.

[3] M. Bordemann. Sur l'existence d'une prescription d'ordre naturelle projectivement invariante. Submitted for publication, math.DG/0208171.

[4] Sofiane Bouarroudj. Projectively equivariant quantization map. Lett. Math. Phys., 51(4):265-274, 2000.

[5] Sofiane Bouarroudj. Formula for the projectively invariant quantization on degree three. C. R. Acad. Sci. Paris Sér. I Math., 333(4):343-346, 2001.

[6] A. Čap, J. Slovák, and V. Souček. Invariant operators on manifolds with almost Hermitian symmetric structures. I. Invariant differentiation. Acta Math. Univ. Comenian. (N.S.), 66(1):33-69, 1997.

[7] A. Čap, J. Slovák, and V. Souček. Invariant operators on manifolds with almost Hermitian symmetric structures. II. Normal Cartan connections. Acta Math. Univ. Comenian. (N.S.), 66(2):203-220, 1997.

[8] C. Duval, P. Lecomte, and V. Ovsienko. Conformally equivariant quantization: existence and uniqueness. Ann. Inst. Fourier (Grenoble), 49(6):1999-2029, 1999.

[9] C. Duval and V. Ovsienko. Projectively equivariant quantization and symbol calculus: noncommutative hypergeometric functions. Lett. Math. Phys., 57(1):61-67, 2001.

[10] Sarah Hansoul. Existence of natural and projectively equivariant quantizations. Submitted for publication, math.DG/0601518.

[11] Sarah Hansoul. Projectively equivariant quantization for differential operators acting on forms. Lett. Math. Phys., 70(2):141-153, 2004.

[12] James Hebda and Craig Roberts. Examples of Thomas-Whitehead projective connections. Differential Geom. Appl., 8(1):87-104, 1998.

[13] James E. Humphreys. Introduction to Lie algebras and representation theory, volume 9 of Graduate Texts in Mathematics. Springer-Verlag, New York, 1978. Second printing, revised.

[14] Shoshichi Kobayashi. Transformation groups in differential geometry. SpringerVerlag, New York, 1972. Ergebnisse der Mathematik und ihrer Grenzgebiete, Band 70 .

[15] P. B. A. Lecomte and V. Yu. Ovsienko. Projectively equivariant symbol calculus. Lett. Math. Phys., 49(3):173-196, 1999.

[16] Pierre B. A. Lecomte. Classification projective des espaces d'opérateurs différentiels agissant sur les densités. C. R. Acad. Sci. Paris Sér. I Math., 328(4):287-290, 1999.

[17] Pierre B. A. Lecomte. Towards projectively equivariant quantization. Progr. Theoret. Phys. Suppl., (144):125-132, 2001. Noncommutative geometry and string theory (Yokohama, 2001).

[18] Pierre Mathonet and Fabian Radoux. Natural and projectively equivariant quantiations by means of cartan connections. Lett. Math. Phys., 72(3):183-196, 2005.

[19] Craig Roberts. The projective connections of T. Y. Thomas and J. H. C. Whitehead applied to invariant connections. Differential Geom. Appl., 5(3):237-255, 1995.

[20] Craig Roberts. Relating Thomas-Whitehead projective connections by a gauge transformation. Math. Phys. Anal. Geom., 7(1):1-8, 2004.

[21] Tracy Yerkes Thomas. A projective theory of affinely connected manifolds. Math. Z., 25:723-733, 1926.

[22] H. Weyl. Zur infinitesimalgeometrie;einordnung der projektiven und der konformen auffassung. Göttingen Nachr., pages 99-122, 1921.

[23] J. H. C. Whitehead. The representation of projective spaces. Ann. of Math. (2), $32(2): 327-360,1931$. 
[24] N. M. J. Woodhouse. Geometric quantization. Oxford Mathematical Monographs. The Clarendon Press Oxford University Press, New York, second edition, 1992. Oxford Science Publications. 